\title{
A New Digital Control DC-DC Converter with Multi-layer Neural Network Predictor
}

\author{
Fujio Kurokawa $^{\dagger}$, Hidenori Maruta $^{\dagger}$, Tomoyuki Mizoguchi $^{\dagger}$, \\ Akihiro Nakamura ${ }^{\dagger}$ and Hiroyuki Osuga ${ }^{\ddagger}$ \\ $\dagger$ Nagasaki University, 1-14 Bunkyo-machi, Nagasaki, 852-8521, Japan \\ † Mitsubishi Electronic Corporation, 325 Kamimachiya, Kamakura, Kanagawa, 247-8520, Japan \\ E-mail: fkurokaw@nagasaki-u.ac.jp
}

\begin{abstract}
The purpose of this paper is to present a new digital control method of the forward type multiple-output dc-dc converter with both a P-I-D feedback and a new feed forward control. In this converter, two novel control methods are proposed. The first new control method is a model method and the second new method is a neural network predictor. The dynamic characteristics of digital control dc-dc converter are improved as compared with the conventional one. Especially, the digital control dc-dc converter with method of the neural network can be realized excellent dynamic characteristics. As a result, the undershoot of the output voltage and the overshoot of reactor current are improved to $45 \%$ and $26 \%$, respectively.
\end{abstract}

\section{Introduction}

The concern with saving the energy has been growing in the world. In the electronics, telecommunications and data communications systems, it has been proposed to introduce the power management function. In the power supply with the power management function, the output power is always changed widely, for example, from a low power sleep operation mode to a high power active operation mode. Therefore, in these areas, the power supply system requires not only the high energy management function but also the high performance dynamic characteristics. Moreover, the high reliability and the small size are necessary. In order to correspond to these requirements, the digital control techniques have been growing to apply to the switching power supply [1]-[6]. In the control method, the P-I-D control method, FIR (Finite Impulse Response) filter method and IIR (Infinite Impulse Response) filter method have been widely used.

So, these control methods have been reported [3]-[7].
This paper presents a new digital control forward type multiple-output dc-dc converter. In this converter, two new control methods are proposed, and the P-I-D control is used as the feedback control and two new control methods are corresponding to the feedforward control, respectively. The first new control method is a model method [7] and the second new method is a machine learning predictor [8],[9]. In the model method, the value of the feedforward control element is changed against the change of the output load. We already reported that the reference value of the output voltage is changed by the model [7]. However, in this method, the circuit becomes unstable under some condition. Therefore, the new model method is presented in this paper. The relational equation between the output load and the time ratio of the main switch are preset and then calculated in the proposed control circuit. In the neural network based method, the value of the feedforward control element is changed by both the change of the output load and predicted data from the neural network, which is widely used in the machine learning area. After reviewing the fundamental configurations of the conventional P-I-D and two new digitally controlled $\mathrm{dc}-\mathrm{dc}$ converters and these operation principles, the dynamic characteristics of three digital control methods are examined. As a result, it is revealed that two new converters have a superior transient response compared with the conventional P-I-D controlled one. Especially, the most excellent dynamic characteristics can be realized in the digital control dc-dc converter with method of the neural network. The key point of this paper is to describe the possibility of realizing a next generation high performance digitally controlled switching power converter with the advanced method of machine learning.

\section{Operation Principle}

Figure 1 shows a new digital control forward type multiple-output dc-dc converter. In the circuit, the reset 
winding $N_{p 2}$ is added to avoid the saturated flux. The turn ratio $N_{p 1} / N_{p 2}$ is equal to unity. Ei is the input voltage, $e_{o 1}$ and $e_{o 2}$ are the output voltages, respectively. $i_{o 1}$ and $i_{o 2}$ are the output currents. $i_{L 1}$ and $i_{L 2}$ are the reactor currents. $D_{11}, D_{12}, D_{21}$ and $D_{22}$ are the diode. $C_{1}$ and $C_{2}$ are the output smoothing capacitor. $N_{p 1}, N_{p 2}, N_{s 1}$ and $N_{s 2}$ are the numbers of turn for the transformer $T . R_{1}$ and $R_{2}$ are the load. $L$ is energy storage reactor with the cross regulation function [10],[11], and $N_{L 1}$ and $N_{L 2}$ are the number of turn for energy storage reactor $L$. The output voltage $e_{o 1}$ is detected and controlled. The output voltage is controlled by the cross regulation of the transformer $T$ and reactor $L$. Particularly, the output currents $i_{o 1}$ is detected as the voltage es by a sensing resistor $R_{s}$ and the input voltage $E_{i}$ is also detected.

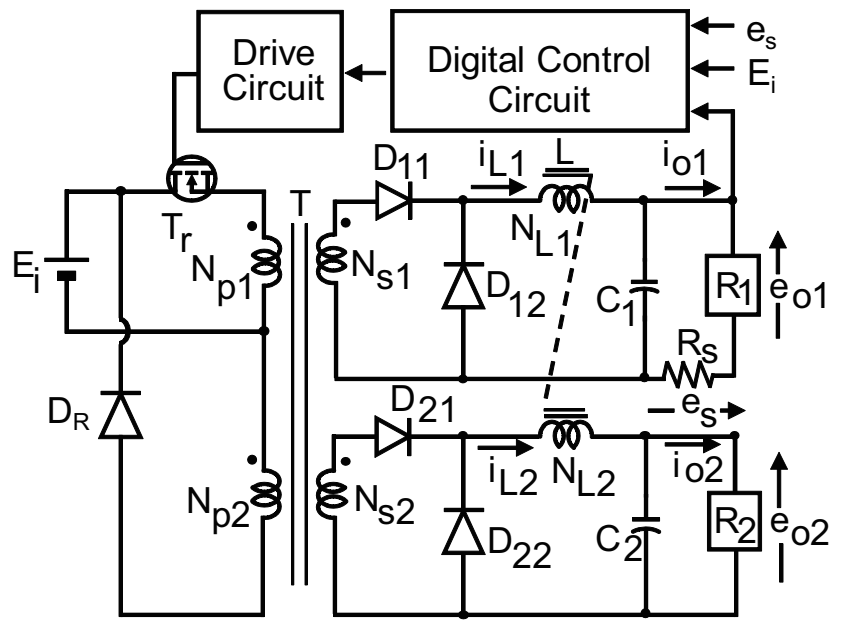

Figure 1. A new digital control multiple-output dc-dc converter.

Figures 2(a) and (b) show the configuration of the proposed digital control circuits. The function of this controller is divided into the P-I-D controller and either the model controller or the neural network predictor. Procedures of the model controller and the neural network predictor are shown in Figs. 3(a) and (b) respectively.

In the P-I-D controllers, the output voltage $e_{o 1}$ of the dc-dc converter is input to the A-D converter through a preamplifier circuit, and converted to the $N_{e o 1}$. In this case, the suffix $\mathrm{n}$ denotes the $\mathrm{n}$-th period of the switching period $T_{S}$. The value is sent to the P-I-D controller and the model controller. Similarly, the input voltage $E_{i}$ and output current $i_{o 1}$ are sent to the model controller.

In the P-I-D controller, the following equation is calculated and the numerical value $N_{\text {Ton_c }}$ corresponded to the on-time from the P-I-D controller is sent to the sutbractor.

$N_{\text {Ton_c }, n}=K_{P}\left(N_{e o, n-2}\right)+K_{I} \sum N_{I, n-2}+K_{D} N_{D, n-2}$

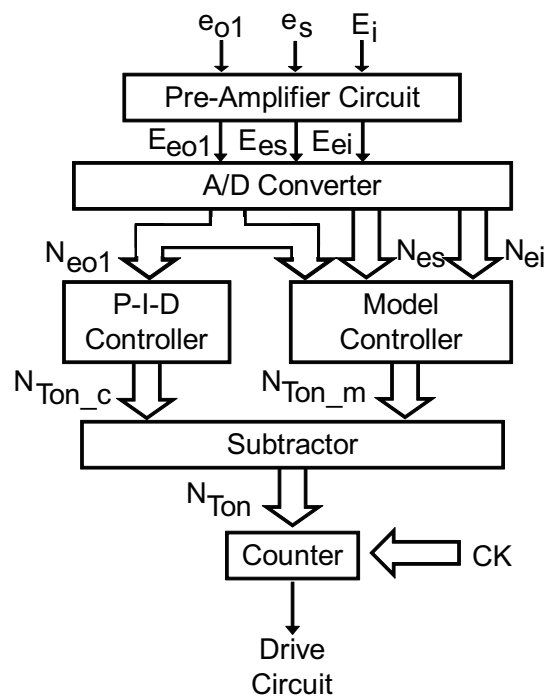

(a)Model based method of feed forward control.

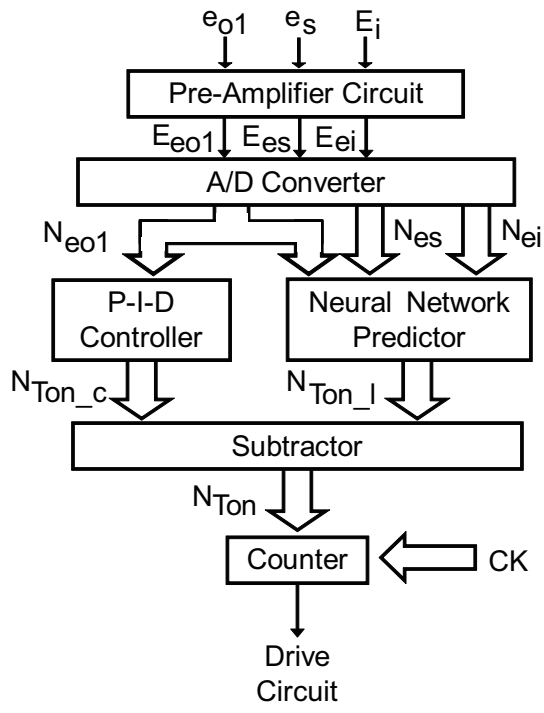

(b)Neural Network based method of feed forward control.

Figure 2. Proposed digital control circuits.

$N_{R}$ is the numerical reference value and $K_{P}$ is the proportional coefficient, respectively. $N_{D, n-1}$ is given by the deference between $N_{e o 1, n-1}$ and $N_{e o 1, n-2} . \quad N_{D, n-1}$ is multiplied by the differential coefficient $K_{D}$ and $K_{D} N_{D, n-1}$ is generated at the multiplier. $\sum N_{I, n-1}$ is given by the integral deference between $N_{e o 1, n-1}$ and $N_{I N T}$. In this case, $N_{I N T}$ is the predetermined reference value in the I-control and corresponds to the desired output voltage of the dc-dc converter. $\sum N_{I, n-1}$ is also multiplied by the integral coefficient $K_{I}$.

In the model controller in Fig. 2 (a), the numerical values 


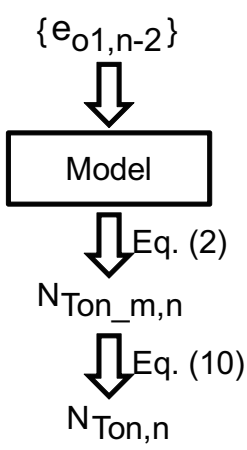

(a)Model based.
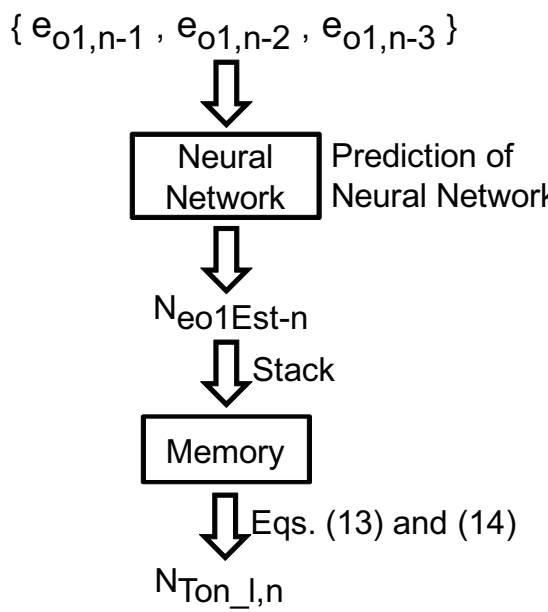

(b)Neural Network based.

\section{Figure 3. Calculation of the feed forward term.}

corresponded to the on-time from the model controller are given by the following equations;

$$
\begin{gathered}
N_{\text {Ton_m, } n}=\frac{\left(1+r_{1} / R_{1}\right) E_{o 1}^{*}}{N_{s 1} / N_{p 1} b-r_{p}\left(a+I_{o 2}\right)} N_{T s}+\frac{a L_{1} N_{T s}}{N_{s} / N_{p 1} b T s} \\
a=N_{e s, n-2} / A_{e s} G_{A D} R_{s} \\
b=N_{e i, n-2} / A_{e s} G_{A D} \\
r_{p}=r_{T r}+r_{T} \\
r_{1}=r_{L 1}+R_{s}
\end{gathered}
$$

The resistance $R_{1}$ is calculated by the sensed output voltage and output current. In Eqs. 1, 3 and 4, $N_{e o 1, n-1}$, $N_{e s, n-1}$ and $N_{e i, n-1}$ are represented as follows;

$$
N_{e o 1, n-2}=A_{e o 1} G_{A D} e_{o 1}
$$

$$
\begin{gathered}
N_{e s, n-2}=A_{e s} G_{A D} R_{s} i_{o 1} \\
N_{e i, n-2}=A_{e i} G_{A D} E_{i}
\end{gathered}
$$

$A_{e s}$ and $G_{A D}$ are the gains of the pre-amplifier and AD converter which sense the output current, and $A_{e o 1}$ is the gains of the pre-amplifier which sense the output voltage, respectively. $N_{\text {Ton_m }}$ is performed as the feedforward control element, as shown in Fig. 3 (a). $N_{\text {Ton_c }}$ and $N_{\text {Ton_m }}$ are sent to the subtractor and the modified $N_{T o n}$ are represented as follows;

$$
\begin{aligned}
N_{\text {Ton }, n}= & N_{\text {Ton } \_m, n}-N_{\text {Ton } \_c, n} \\
= & N_{\text {Ton } \_m, n}-\left\{K_{P}\left(N_{e o 1, n-2}-N_{R}\right)\right. \\
+ & \left.K_{I} \sum N_{I, n-1}+K_{D}\left(N_{e o 1, n-2}-N_{n-2}\right)\right\} \\
& \quad T_{o n, n}=\left(N_{\text {Ton }, n} / N_{T s}\right) T_{s}
\end{aligned}
$$

In the neural network approach illustrated in Fig. 2(b), to control $N_{T o n, n}$, a multi-layer neural network method is applied as a feedforward controller. Notice that P-I-D controller is remained, the neural network controller is considered as the predictor of non-linear dynamical system. In this proposed method, three-layer neural network is applied. This neural network predicts $e_{o 1 E s t, n}$, the n-th value of output voltage $e_{o 1, n}$, using its 3 former data, $e_{o 1, n-1}, e_{o 1, n-2}$, $e_{o 1, n-3}$ as shown in Fig. 3 (b). The number of the unit of input layer becomes three and the number of the hidden unit is set six, twice number of the input layer's unit in this case. By definition, the output layer become one unit, which is predicted $e_{o 1, n}$. In this neural network, sigmoid function is used as the activation function. Weight parameters are randomly initialized and are trained by back propagation algorithm with the standard sum-of-squares error function. To train this neural network, one periodic data of $e_{o 1}$ without the neural network control, which are obtained from conventional digital control circuits, is used as the learning data. In this case, the number of data points is 1000 due to the switching frequency. After iterations (1000 times in this case) with the back propagation algorithm using this learning data, the obtained neural network controller is considered as the predictor of $e_{o 1, n}$. After $e_{o 1 E s t, n}$, which is the predicted value of $e_{o 1, n}$, is predicted, the feedforward controller term $N_{e o 1 E s t, n}$ is obtained. The numerical value $N_{T o n, n}$ with neural network controller corresponded to the on-time is represented as follows;

$$
\begin{aligned}
N_{\text {Ton }, n} & =N_{\text {Ton_1 }, n}-N_{\text {Ton_c }, n} \\
& =N_{\text {Ton_1 }, n}-\left\{K_{P}\left(N_{e o 1, n-2}-N_{R}\right)\right. \\
& \left.+K_{I} \sum N_{I, n-1}+K_{D}\left(N_{e o 1, n-2}-N_{n-2}\right)\right\}
\end{aligned}
$$

$$
N_{\text {Ton_ } 1, n}=\alpha_{n}\left(N_{e o 1, n-1}-N_{e o 1 E s t, n}\right)
$$




$$
\alpha_{n}=A \cdot e^{-\lambda_{n}}
$$

where $N_{e o 1, n-1}$ is obtained by substitution of $e_{o 1}$ into Eq. 7 and it is numerical desired output voltage corresponded to the desired output voltage. Further, $N_{e o 1 E s t, n}$ is obtained by substitution of the estimated $e_{o 1, n}$ with the neural network controller into Eq. 7. $A$ and $\lambda$ are the coefficients, respectively. Notice that $n$ denotes the n-th switching period from the point in which the step response of the load is occurred. Once $\left\{N_{e o 1 E s t, n}\right\}$ are obtained, they are stacked into the memory as shown in Fig. 3 (b). The $\left\{N_{e o 1 E s t, n}\right\}$ are performed as the feedforward control elements with nodelay. When the change of load is occurred, the resistance is calculated by the sensed output voltage and output current. In the neural network predictor, the predict value $N_{o 1 E s t, n}$ is generated about each load $R$ at the previous and current time points.

\section{Transient Response}

Figures 4 through 6 show the simulated transient response of the conventional P-I-D control and two new digital control method of the forward type multiple-output dcdc converter in step change of the load resistor $R_{1}$ from $25 \Omega$ to $2.5 \Omega$. In this case, $R_{2}$ is constant. In the conventional P-I-D control dc-dc converter, the sensing resistor $R_{s}$ is removed because the output current $i_{o 1}$ is not detected. The simulator is PSIM. The switching frequency is $200 \mathrm{kHz}$. The circuit parameters are $E_{i}=36 \mathrm{~V}, E_{o 1}^{*}=5 \mathrm{~V}$, $C_{1}=C_{2}=1,000 \mu F, R_{S}=0.001 \Omega, A_{e o 1}=0.5$, $A_{e s}=100, A_{E i}=0.0625$ and $G_{A D}=819$. The proportional coefficient $K_{P}$ is 5 , the integral coefficient $K_{I}$ is 0.024 and the differential coefficient $K_{D}$ is 2 . The number of bit of A-D converter is 12. Figure 4 shows that the undershoot, overshoot and transient time of the output voltage $e_{o 1}$ are $1.8 \%, 0.8 \%$ and $0.21 \mathrm{~ms}$ in the conventional P-I-D control method. The convergence time tst that the output voltage $e_{o 1}$ is settled within $1 \%$ is $0.21 \mathrm{~ms}$. The overshoot of reactor current $i_{L 1}$ is $39 \%$. Figure 5 shows the simulated transient response of the proposed model control method. The digital control circuit parameters are $K_{P}=2$, $K_{I}=0.00001$ and $K_{D}=2$, respectively. These parameters are selected to optimize the dynamical characteristics of the transient response for this method.

The overshoot and undershoot of the output voltage $e_{o 1}$ is over $2.2 \%, 0.7 \%$. The convergence time $t_{s t}$ is $0.34 \mathrm{~ms}$. The overshoot of reactor current $i_{L 1}$ is $28 \%$.

Figure 6 shows the simulated transient response according to the neural network based control method of the forward type multiple-output dc-dc converter. The digital control circuit parameters are same as above conventional P-ID control method. $A$ and $\lambda$ are set 85 and 40,000, respectively. The coefficient $A$ is very important to suppress the
Table 1. Summary of conventional and proposed methods about output voltage $\mathrm{e}_{\mathrm{o} 1}$.

\begin{tabular}{|c|c|c|c|}
\hline & P-I-D & Model & Neural Network \\
\hline undershoot(\%) & 1.8 & 2.2 & 1.0 \\
\hline overshoot $(\%)$ & 0.8 & 0.7 & 0.4 \\
\hline$t_{s t}(\mathrm{~ms})$ & 0.21 & 0.34 & 0.08 \\
\hline
\end{tabular}

Table 2. Summary of conventional and proposed methods about reactor current $i_{L 1}$.

\begin{tabular}{|c|c|c|c|}
\hline & P-I-D & Model & Neural Network \\
\hline overshoot(\%) & 39 & 28 & 29 \\
\hline
\end{tabular}

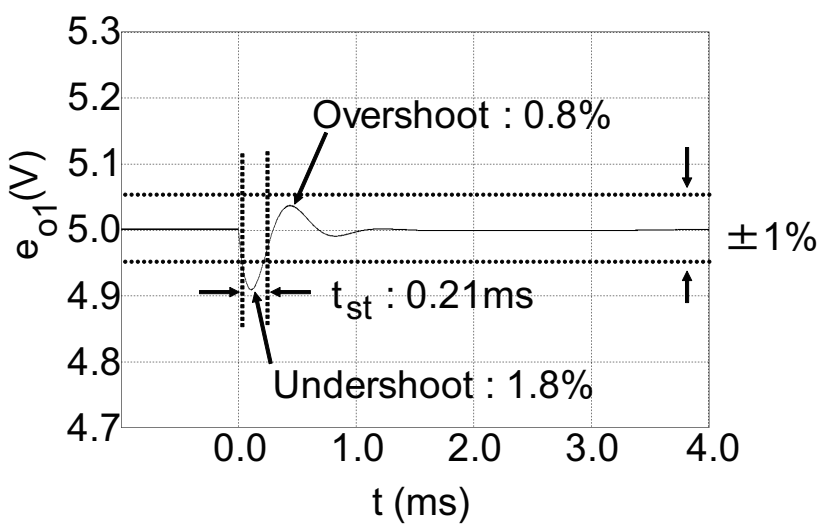

(a) Output voltage $e_{o 1}$.

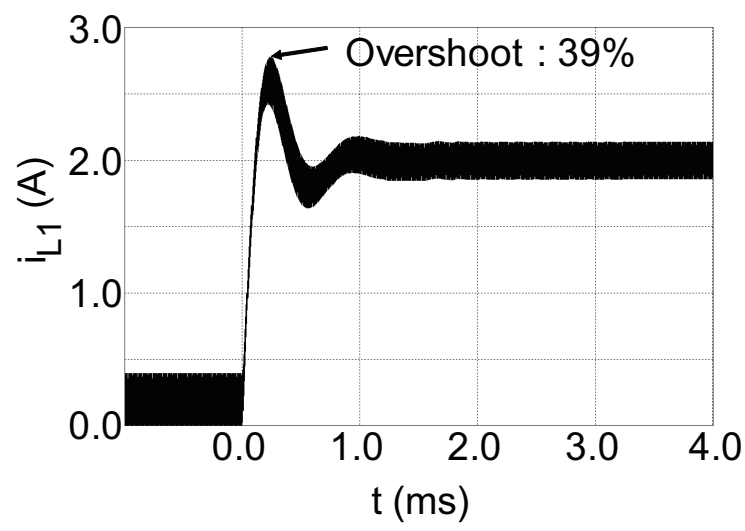

(b) Reactor current $i_{L 1}$

Figure 4. Transient response of conventional P-I-D control. 


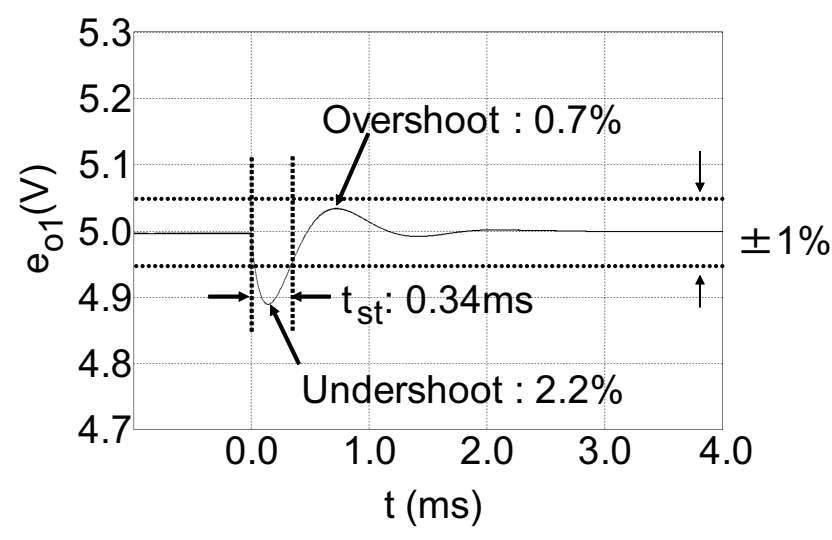

(a) Output voltage $e_{o 1}$.

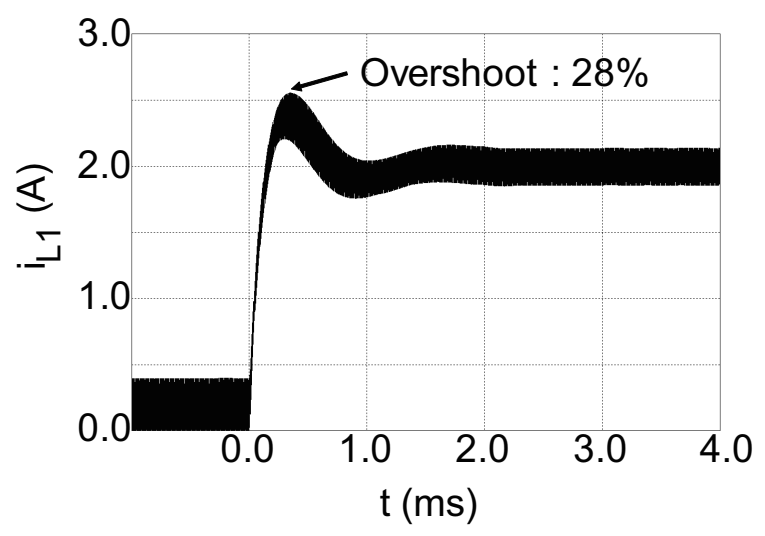

(b) Reactor current $i_{L 1}$

\section{Figure 5. Transient response using proposed model controller.}

undershoot of output voltage $e_{o 1}$ and the overshoot of reactor current, the coefficient $\lambda$ is also performed to suppress the overshoot of the output voltage $e_{o 1}$.

The overshoot and undershoot of the output voltage $e_{o 1}$ is under $0.4 \%$ and $1.0 \%$ respectively in case of a neural network based control. The convergence time $t_{s t}$ is less than $0.08 \mathrm{~ms}$. The overshoot of reactor current $i_{L 1}$ is under $29 \%$. The undershoot of the output voltage, the overshoot of reactor current $i_{L 1}$ and the convergence time tst are improved to $45 \%, 26 \%$ and $62 \%$, respectively.

As a result, it is revealed that the transient response of the dc-dc converter is improved by adding the neural network based control as the feedforward control.

\section{Conclusion}

The transient response to step change of the load is discussed in a new digital control method of the forward type

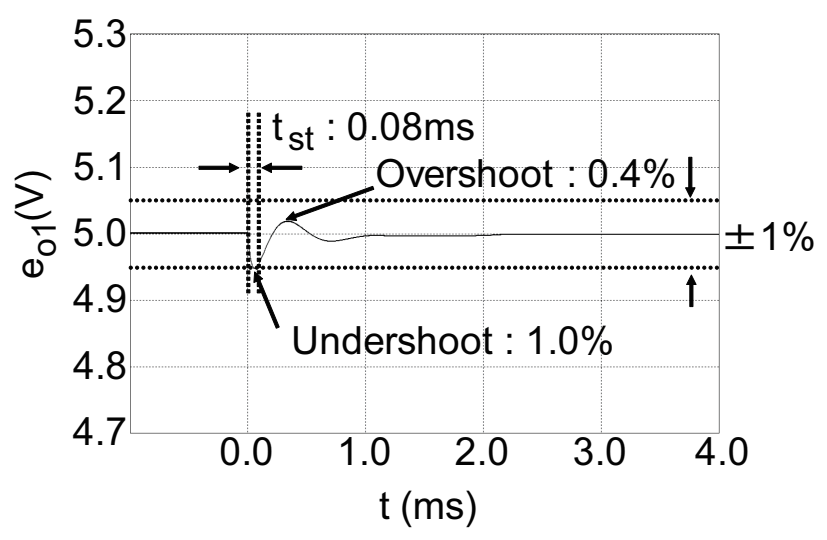

(a)Output voltage $e_{o 1}$.

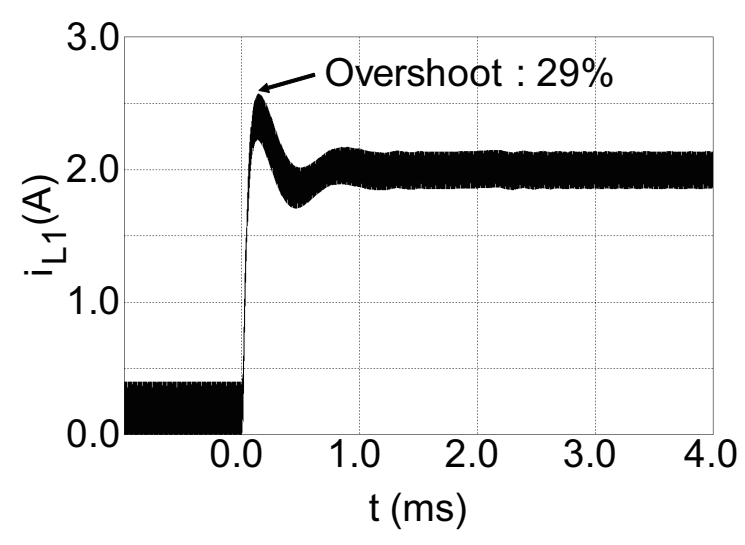

(b)Reactor current $i_{L 1}$

Figure 6. Transient response using proposed neural network predictor.

multi-output dc-dc converter.

It seems that excellent characteristic is otbained in the proposed P-I-D control method adding the neural network predictor as the feedforward controller. It is clarified that the undershoot of the output voltage is $1 \%$ and the convergence time that the output voltage is settled within $1 \%$ is $0.08 \mathrm{~ms}$. The overshoot of reactor current is $29 \%$. The effect of the neural network predictor is superior than that of the model controller. The undershoot of output voltage of the neural network method is less than one half of that of model method. The convergence time is one quarter. Namely, the undershoot of the output voltage, the overshoot of reactor current and convergence time are widely improved to $45 \%, 26 \%$ and $62 \%$, respectively, compared with the conventional P-I-D control method. It is confirmed that a new digital control method of the forward type multi-output dcdc converter is useful to realize the high performance digital control circuit of dc-dc converter. 
This work is supported in part by the Grant-in-Aid for Scientific Research (No.21360134) of JSPS (Japan Society for the Promotion of Science) and the Ministry of Education, Science, Sports and Culture.

\section{References}

[1] D. Maksimovic, R. Zane and R. Erickson, "Impact of digital control in power electronics," Proceedings of International Symposium on Power Semiconductor Devices \& ICs, pp. 13-22, May 2004.

[2] P. T. Krein, "Digital control generations -digital controls for power electronics through the third generation," Proceedings of the IEEE International Conference on Power Electronics and Drive Systems, November 2007.

[3] L. Guo, J. Y. Hung and R. M. Nelms, "PID controller modifications to improve steady-state- performance of digital controllers for buck and boost converters," Proceedings of Annual IEEE Applied Power Electronics Conference, no. 9.3, pp. 381-388, March 2002.

[4] W. Stefanutti, S. Saggini, E. Tedeschi, P. Mattavelli and P. Tenti, "Simplified model reference tuning of PID regulators of digitally controlled dc-dc converters based on crossover frequency analysis," IEEE Power Electronics Specialists Conference Record, pp.785791, June 2007.

[5] F. Kurokawa, M. Okamatsu, T. Ishibashi, and Y. Nishida, "Dynamic characteristics of dc-dc converters using digital filters," Journal of Power Electronics, vol. 9, no. 3, pp. 430-437, May 2009.

[6] F. Kurokawa and M. Okamatsu, "Static and dynamic characteristics of dc-dc converter using a digital filter," IEICE Trans. Commun., vol. E92-B, no. 3, pp. 9981003, March 2009.

[7] F. Kurokawa and S. Sukita, "A new model control dc-dc converter to improve dynamic characteristics," Proceedings of the IEEE International Conference on Power Electronics and Drive Systems, pp. 763-767, Nov. 2007.

[8] T. Masters, "Practical Neural Network Recipes in C++," Morgan Kaufmann, 1995.

[9] T. Hastie, R. Tibshirani, J.H. Friedman, "The elements of statistical learning: data mining, inference, and prediction," Springer, 2001.

[10] H. Matsuo and F. Kurokawa: "Analysis of multipleoutput dc-dc power converter using cross-regulation,"
Trans. IECEof Japan, vol. 62-C, no. 8, pp. 550-557, August 1979.

[11] H. Saotome, S. Oikawa, Y. Kikuchi, N. Sekino and M. Hayashi: "Analysis of cross-regulation in multipleoutput DC/DC converters," Trans. IEICE of Japan, vol. 104, no. 407, pp. 25-30, Nov 2004. 